\title{
The MOLEN Polymorphic Processor
}

\author{
Stamatis Vassiliadis, Fellow, IEEE, Stephan Wong, Member, IEEE, \\ Georgi Gaydadjiev, Member, IEEE, Koen Bertels, Member, IEEE, \\ Georgi Kuzmanov, Student Member, IEEE, and Elena Moscu Panainte
}

\begin{abstract}
In this paper, we present a polymorphic processor paradigm incorporating both general purpose and custom computing processing. The proposal incorporates an arbitrary number of programmable units, exposes the hardware to the programmers/ designers, and allows them to modify and extend the processor functionality at will. To achieve the previously stated attributes, we present a new programming paradigm, a new instruction set architecture, a microcode-based microarchitecture, and a compiler methodology. The programming paradigm, in contrast with the conventional programming paradigms, allows general-purpose conventional code and hardware descriptions to coexist in a program. In our proposal, for a given instruction set architecture, a onetime instruction set extension of eight instructions is sufficient to implement the reconfigurable functionality of the processor. We propose a microarchitecture based on reconfigurable hardware emulation to allow high-speed reconfiguration and execution. To prove the viability of the proposal, we experimented with the MPEG-2 encoder and decoder and a Xilinx Virtex II Pro FPGA. We have implemented three operations, SAD, DCT, and IDCT. The overall attainable application speedup for the MPEG-2 encoder and decoder is between 2.64-3.18 and between 1.56-1.94, respectively, representing between 93 percent and 98 percent of the theoretically obtainable speedups.
\end{abstract}

Index Terms-Custom computing machines, FPGA, firmware, reconfigurable microcode, polymorphic processors, reconfigurable processors.

\section{INTRODUCTION}

G ENERAL-PURPOSE processors allow us to run the same J program over a range of implementations of the same architectural family [1] in a compatible manner. Furthermore, they allow various programs to run on the same system and the same program to run over multiple processing families. One of the major continuous concerns of general-purpose processors is performance. Reconfigurable hardware coexisting with a core processor has been considered a good candidate to address such a concern. Even though such an approach is promising and several processor paradigms have been proposed, see numerous examples in [2], [3], the organization of such a hybrid processor can be viewed mostly as an open topic. In this paper, we propose a polymorphic processor that substantially improves various aspects, including performance, of such a hybrid general purpose processor paradigm. The main contributions of the proposed approach can be summarized by the following:

- For a given ISA, a one-time architectural extension (based on the coprocessor architectural paradigm) comprised of eight instructions suffices to provide an almost arbitrary number of reconfiguration "functions" per single programming space. This realization resolves the opcode space explosion and modularity problems and provides ISA compatibility

- The authors are with the Computer Engineering Laboratory, Delft University of Technology, Mekelweg 4, 2628CD Delft, The Netherlands. E-mail: \{S.Vassiliadis, J.S.S.M.Wong, G.N.Gaydadjiev, K.L.M.Bertels, G.Kuzmanov\}@ewi.tudelft.nl, elena@ce.et.tudelft.nl.

Manuscript received 10 Dec. 2003; revised 10 Apr. 2004; accepted 16 Apr. 2004.

For information on obtaining reprints of this article, please send e-mail to: tc@computer.org, and reference IEEECS Log Number TCSI-0278-1203. and portability of reconfigurable programs, present in previous proposals, such as the ones described in [4], [5], [6].

- We propose a new processor organization and we describe a programming paradigm based on sequential consistency that allows the proposed coprocessor environment to coexist with the general-purpose processor and to resolve parameter limitations and parallel execution problems, present in other proposals (see, for example, [7], [8]).

- We propose a back-end compiler technology that allows us to target the proposed processor architecture, a microarchitecture based on reconfigurable emulation ( $\rho \mu$-code), and an implementation that allows the compiled code to execute.

The paper is organized as follows: Section 2 discusses related work and describes the general approach of how to modify an existing program to support reconfigurable computing. Section 3 introduces the Molen organization, the Molen programming paradigm, and the polymorphic instruction set architecture ( $\pi \mathrm{ISA})$. Section 4 discusses the sequencing and compiler extensions required to implement the Molen programming paradigm. Section 5 describes in detail the underlying microarchitecture and the $\rho \mu$-code unit. Section 6 presents an evaluation of the proposed Molen architecture. Section 7 presents the overall conclusions.

\section{Related Work and General Approach}

As indicated earlier, reconfigurable hardware coexisting with a core general-purpose processor has been considered by several researchers as a good candidate for speeding up applications. For the description of most of the existing proposals, the interested reader is referred to two review/classification articles [2], [3]. Current 


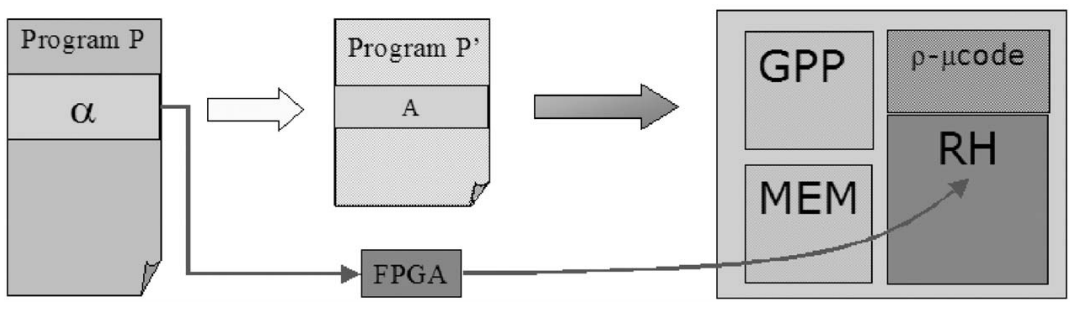

Fig. 1. Program transformation example.

reconfigurable computing proposals, where the possibility exists to combine general-purpose computing with reconfigurable fabric, fall short of expectation because of the following shortcomings:

- Opcode space explosion. For reconfigurable fabric, a common approach (e.g., [4], [5], [6]) is to introduce a new instruction for each portion of the application mapped on the field-programmable gate array (FPGA). The consequence is the limitation of the number of operations implemented on the FPGA, due to the limitation of the opcode space. More specifically stated, for a specific application domain intended to be implemented on the FPGA, the designer and compiler are restricted by the unused opcode space. Furthermore, this results in ad hoc instruction set architecture (ISA) extensions, which excludes compatibility.

- No modularity. Each approach has a specific definition and implementation bounded for a specific reconfigurable technology and design. Consequently, the applications cannot be (easily) ported to a new reconfigurable platform. Further, there are no mechanisms allowing reconfigurable implementations to be developed separately and ported transparently, as indicated in [9]. This implies that a reconfigurable implementation developed by vendor A cannot be included without substantial effort by the compiler developed for an FPGA implementation provided by vendor $\mathrm{B}$.

Additional shortcomings of current proposals regarding performance gains include the following:

- Limitation of the number of parameters. In a number of approaches, the operations mapped on an FPGA can only have a small number of input and output parameters (e.g., [7], [8]). For example, in the architecture presented in [7], due to the encoding limits, the fragments mapped onto the FPGA have at most four inputs and two outputs; also, in [8], the maximum number of input registers is nine and it has one output register.

- No support for parallel execution on the FPGA of sequential operations. An important and powerful feature of FPGAs can be the parallel execution of sequential operations when they have no data dependencies. Many architectures (see, for example, [2]) do not take into account this issue and their mechanism for FPGA integration cannot be extended to support parallelism.

In the discussion to follow, we present the general concept of transforming an existing program to one that can be executed on the reconfigurable computing platform we propose and hints to the new mechanisms, intended to improve existing approaches.

The conceptual view of how program $\mathrm{P}$ (intended to execute only on the general-purpose processor (GPP) core) is transformed into program $\mathrm{P}^{\prime}$ (executing on both the GPP core and the reconfigurable hardware) is depicted in Fig. 1. The purpose is to obtain a functionally equivalent program $\mathrm{P}^{\prime}$ from program $\mathrm{P}$ which (using specialized instructions) can initiate both the configuration and execution processes on the reconfigurable hardware. The steps involved in this transformation are the following:

1. Identify code " $\alpha$ " in program $\mathrm{P}$ to be mapped in reconfigurable hardware.

2. Show that " $\alpha$ " can be implemented in hardware in an existing technology, e.g., FPGA, and map " $\alpha$ " onto reconfigurable hardware $(\mathrm{RH})$.

3. Eliminate the identified code " $\alpha$ " and add "equivalent" code (A) assuming that code A "calls" the hardware with functionality " $\alpha$. " Code A is comprised of the following:

- Repair code inserted to communicate parameters and results to/from the reconfigurable hardware from/to the general-purpose processor core.

- "HDL"-like hardware code and emulation code inserted to configure the reconfigurable hardware and to perform the functionality that is initialized by the "execute code".

4. Compile and execute program $\mathrm{P}^{\prime}$ with original code plus code having functionality A (equivalent to functionality " $\alpha$ ") on the GPP/reconfigurable processor.

The mentioned steps illustrate the need for a new programming paradigm in which both software and hardware descriptions are present in the same program. It should also be noted that the only constraint on " $\alpha$ " is implementability, which possibly implies complex hardware. Consequently, the microarchitecture may have to support emulation [11] via microcode. We have termed this reconfigurable microcode ( $\rho \mu$-code) as it is different from the traditional microcode. The difference is that such a microcode does not execute on fixed hardware facilities. It operates on facilities that the $\rho \mu$-code itself "designs" to operate upon. The methodology of the transformation described previously for the reconfigurable computing platform is depicted in Fig. 2. First, the code to be executed on the reconfigurable hardware must be determined. This is achieved by high-level to high-level instrumentation and benchmarking. This results in several candidate pieces of 


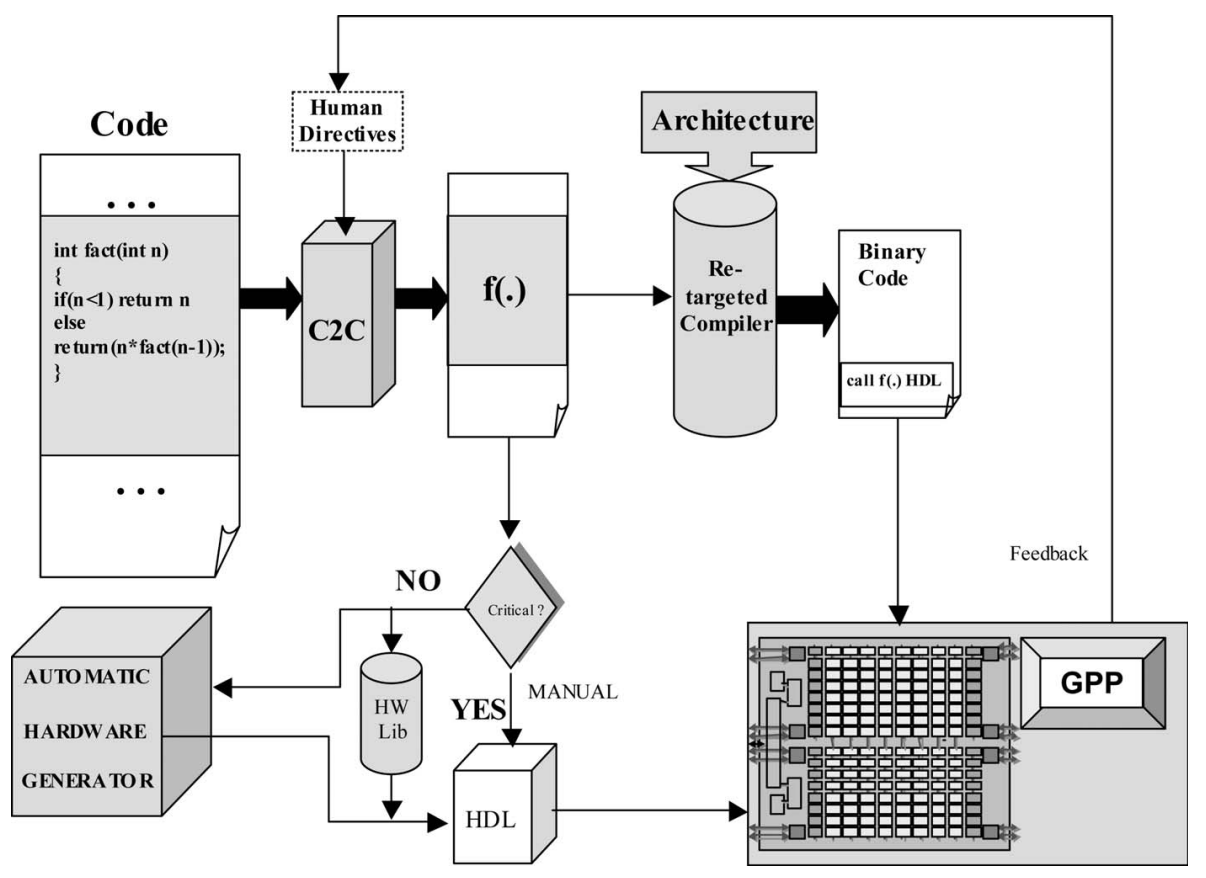

Fig. 2. Program transformation methodology for reconfigurable computing.

code. Second, we must determine which piece of code is suitable for implementation on the reconfigurable hardware. The suitability is solely determined by whether the piece of code is implementable (i.e., "fits in hardware"). Those parts can then be mapped into hardware via a hardware description language (HDL). In case the HDL corresponds to "critical" hardware in terms of, for instance, area, performance, memory, and power consumption, the translation will be done manually (see Fig. 2). Otherwise, the translation can be done automatically, as, for example, described in [10], [12], [13], or be extracted from a library.

\section{Organization, ISA, and Programming}

The two main components in the Molen machine organization [14] (depicted in Fig. 3) are the "Core Processor," which is a general-purpose processor (GPP), and the "Reconfigurable Processor" (RP). Instructions are issued to either processor by the "Arbiter" and data are fetched (stored)

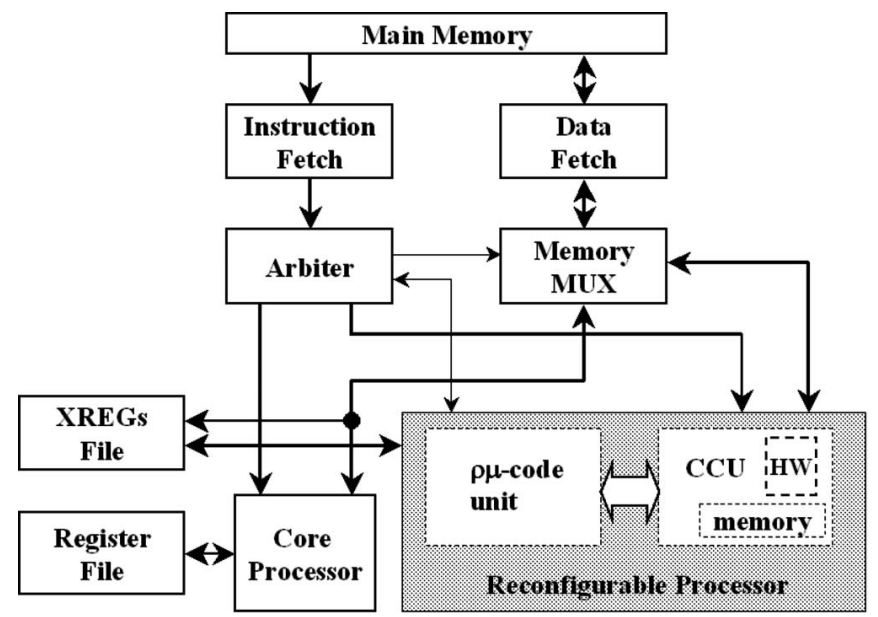

Fig. 3. The Molen machine organization. by the "Data Fetch" unit. The "Memory MUX" unit is responsible for distributing(collecting) data.

The reconfigurable processor is further subdivided into the $\rho \mu$-code unit (discussed in Section 5) and the custom configured unit (CCU). The CCU consists of reconfigurable hardware, e.g., a field-programmable gate array (FPGA), and memory. All code runs on the GPP except pieces of (application) code implemented on the CCU in order to speed up program execution. Exchange of data between the GPP and the RP is performed via the exchange registers (XREGs) (described in Section 4) depicted in Fig. 3. The envisioned support of operations ${ }^{1}$ by the reconfigurable processor can be initially divided into two distinct phases: set and execute. In the set phase, the CCU is configured to perform the supported operations. Subsequently, in the execute phase, the actual execution of the operations is performed. This decoupling allows the set phase to be scheduled well ahead of the execute phase, thereby hiding the reconfiguration latency. As no actual execution is performed in the set phase, it can even be scheduled upward across the code boundary in the code preceding the RP targeted code.

In order to target the $\rho \mu$-code processor, we propose a sequential consistency programming paradigm [15]. The paradigm allows for parallel and concurrent hardware execution and it is intended (currently) for single program execution. It requires only a one-time architectural extension of a few instructions to provide a large user reconfigurable operation space. The complete list of the eight required instructions, denoted as polymorphic

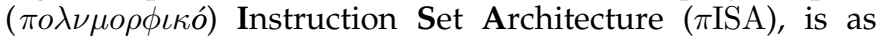
follows:

- Six instructions are required for controlling the reconfigurable hardware, namely:

1. An operation can be as simple as a single instruction or as complex as a piece of code. 
Two set instructions. These instructions initiate the configurations of the CCU. Two instructions are added for partial reconfiguration:

* The partial set $(p$-set $<$ address $>$ ) instruction performs those configurations that cover common parts of multiple functions and/or frequently used functions. In this manner, a considerable number of reconfigurable blocks in the CCU can be preconfigured.

* The complete set $(c$-set $<$ address $>)$ instruction performs the configurations of the remaining blocks of the CCU (not covered by the $p$-set) to complete the CCU functionality.

We must note that, in case no partial reconfigurable hardware is present, the $c$-set instruction alone can be utilized to perform all the necessary configurations.

execute $<$ address $>$. This instruction controls the execution of the operations implemented on the CCU. These implementations are configured onto the CCU by the set instructions.

- $\quad$ set prefetch $<$ address $>$. This instruction prefetches the needed microcode responsible for CCU reconfigurations into a local on-chip storage facility (the $\rho \mu$-code unit) in order to possibly diminish microcode loading times.

- execute prefetch $<$ address $>$. The same reasoning as for the set prefetch instruction holds, but now relating to microcode responsible for CCU executions.

- break. This instruction is utilized to facilitate the parallel execution of both the reconfigurable processor and the core processor. More precisely, it is utilized as a synchronization mechanism to complete the parallel execution.

- Two move instructions for passing values between the register file and exchange registers (XREGs):

- movtx $\mathrm{XREG}_{a} \leftarrow \mathrm{R}_{b}$. The (move to XREG) instruction used to move the content of general-purpose register $\mathrm{R}_{b}$ to $\mathrm{XREG}_{a}$.

- movfx $\mathrm{R}_{a} \leftarrow \mathrm{XREG}_{b}$. The (move from XREG) instruction used to move the content of exchange register $\mathrm{XREG}_{b}$ to general-purpose register $\mathrm{R}_{a}$.

The $<$ address $>$ field in instructions introduced above denotes the location of the reconfigurable microcode responsible for the configuration and execution processes (see Section 5). It must be noted that a single address space is provided with at least $2^{(n-o p)}$ addressable functions for reconfiguration, where $n$ represents the instruction word length and $o p$ the opcode length. If $2^{(n-o p)}$ is found to be insufficient, indirect pointing or GPP-like status word mechanisms can extend the addressing of the reconfigurable function space at will. Code fragments constituted of contiguous statements (as they are represented in high-level programming languages) can be isolated as generally implementable functions (that is, code with multiple identifiable input/output values). The parameters are passed via the exchange registers (XREGs). In order to maintain correct program semantics, the code is annotated and a hardware description file provides the compiler with implementation specific information, such as the addresses, where the reconfigurable microcode is to be stored, the number of exchange registers, etc. It should be noted that it is not imperative to include all instructions when implementing the Molen organization. The programmer/implementor can opt for different ISA extensions depending on the performance that needs to be achieved and the available technology. There are basically three distinctive $\pi \mathrm{ISA}$ possibilities with respect to the Molen instructions introduced earlier-the minimal, the preferred, and the complete $\pi$ ISA extension. In more detail, they are the following:

- The minimal $\pi$ ISA. This is essentially the smallest set of Molen instructions needed to provide a working scenario. The four basic instructions needed are set (more specifically: $c$-set), execute, movtx, and movfx. By implementing the first two instructions (set/execute), any suitable CCU implementation can be loaded and executed in the reconfigurable processor. Furthermore, reconfiguration latencies can be hidden by scheduling the set instruction considerably earlier than the execute instruction. The movtx and movfx instructions are needed to provide the input/output interface between the RP targeted code and the remaining application code.

- The preferred $\pi$ ISA. In order to address reconfiguration latencies both $p$-set and $c$-set instructions are utilized. In this case, as the reconfiguration latencies are substantially (or completely) hidden, the loading time of microcode will play an increasingly important role. In these cases, the two prefetch instructions (set prefetch and execute prefetch) provide a way to diminish the microcode loading times by scheduling them well ahead of the moment that the microcode is needed. Parallel execution, for both minimal and preferred $\pi$ ISA is initiated by a set/execute instruction and ended by a generalpurpose instruction (see Fig. 4a).

- $\quad$ The complete $\pi$ ISA. This scenario involves all $\pi$ ISA instructions including the break instruction. In some applications, it might be beneficial performance-wise to execute instructions on the core processor and the reconfigurable processor in parallel. In order to facilitate this parallel execution, the preferred ISA is further extended with the break instruction. The break instruction provides a mechanism to synchronize the parallel execution of instructions by halting the execution of instructions following the break instruction. The sequence of instructions performed in parallel is initiated by an execute instruction. The end of the parallel execution is marked by the break instruction. It indicates where the parallel execution stops (see Fig. 4b). The set instructions are executed in parallel according to the same rules.

\section{Compiler and Program Sequence Control}

We begin by discussing the exchange registers (XREGs) and the parameter and result passing mechanism between the general-purpose processor and the reconfigurable processor.

The Exchange Registers. The XREGs are used for passing operation parameters to the reconfigurable hardware and returning the computed values after operation 


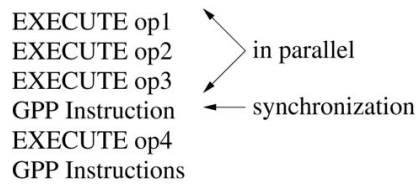

(a)

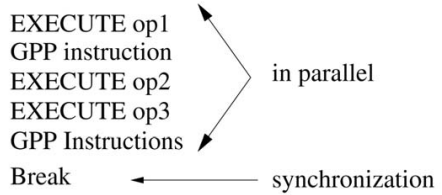

(b)

Fig. 4. Parallel execution and models of synchronization. (a) Synchronization when consecutive EXECUTE instructions are performed in parallel and GPP is stalled (the preferred $\pi$ ISA). (b) Synchronization when GPP and RP work in parallel (the complete $\pi$ ISA).

execution. Parameters are moved from the register file to the XREGs (movtx) and the results stored back from the XREGs in the register file (movfx) and the reconfigurable microcode is responsible for managing the parameters from the XREGs and returning the result(s). The following conventions are introduced for single and parallel execution: All parameters of an operation are allocated by the compiler in consecutive XREGs forming a block of XREGs. The microcode of each execute instruction has a fixed XREG, which has been assigned during the microcode development. The compiler places in this XREG a link to the block of XREGs where all parameters are stored. This link is the number of the first XREG in the block. Based on these conventions, the parameters for all operations can be efficiently allocated by the compiler and the microcode of each execute instruction is able to locate its associated block of parameters. An example is presented in Fig. 5, where two operations, namely, op 1 and op2, are executed in parallel. Their fixed XREGs (XREG0 and XREG1) are communicated to the compiler in a hardware description file. As indicated by the number stored in XREG0, the compiler allocates, for operation op1, two consecutive XREGs for passing parameters and returning results, namely, XREG2 and XREG3. The operation $o p 2$ requires only one XREG for parameters and results passing, which, in the example, is XREG4, as indicated by the content of XREG1.

The Compiler. Currently, the compiler [16] relies on the Stanford SUIF2 [17] (Stanford University Intermediate Format) Compiler Infrastructure for the front-end and for the back-end on the Harvard Machine SUIF [18] framework. The following essential features for a compiler targeting a custom computing machines (CCM) have currently been implemented:

- Code identification. For the identification of the code mapped on the reconfigurable hardware, we added a special pass in the SUIF front-end. This identification

\section{EXECUTE address_op1 EXECUTE address_op2}

op1 : fix XR $\rightarrow$ XR0 op2 : fix XR $\rightarrow$ XR1

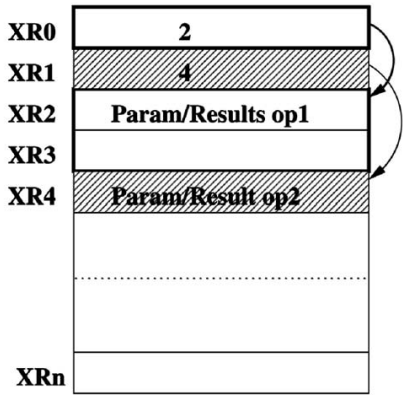

Fig. 5. Exchange Registers allocation by the compiler. is based on code annotation with special pragma directives (similar to [6]). In this pass, all the calls of the recognized functions are marked for further modification.

- Instruction set extension. The instruction set has been extended with set/execute instructions at both the medium intermediate representation level and low intermediate representation (LIR) level.

- Register file extension. The register file set has been extended with the exchange registers. The register allocation algorithm allocates the XREGs in a distinct pass applied before the register allocation; it is introduced in Machine SUIF, at LIR level. The conventions introduced for the XREGs are implemented in this pass.

- Code generation. Code generation for the reconfigurable hardware (as previously presented) is performed when translating SUIF to Machine SUIF intermediate representation and affects the function calls marked in the front-end.

An example of the code generated by the extended compiler for the Molen programming paradigm is presented in Fig. 6. On the left, the $C$ code is depicted. The function implemented in reconfigurable hardware is annotated with a pragma directive named call_fpga. It has incorporated the operation name, op1, as specified in the hardware description file. In the middle, the code generated by the original compiler for the $\mathrm{C}$ code is depicted. The pragma annotation is ignored and a normal function call is included. On the right, the code generated by the compiler extended for the Molen programming paradigm is depicted; the function call is replaced with the appropriate instructions for sending parameters to the reconfigurable hardware in XREGs, hardware reconfiguration, preparing the fixed XREG for the microcode of the execute instruction, execution of the operation, and the transfer of the result back to the general-purpose register file. The presented code is at medium intermediate representation level in which the register allocation pass has not yet been applied.

The compiler extracts from a hardware description file the information about the target architecture such as the microcode address of the set and execute instructions for each operation implemented in the reconfigurable hardware, the number of XREGs, the fixed XREG associated with each operation, etc.

Parameter exchange, parallelism and modularity. As shown earlier, the exchange registers solve the limitation on the number of parameters present in other reconfigurable computing approaches. If the parameters do not exceed the number of XREGs, parameters are passed by value, otherwise -by reference. The Molen architecture also addresses an additional shortcoming of other reconfigurable computing 


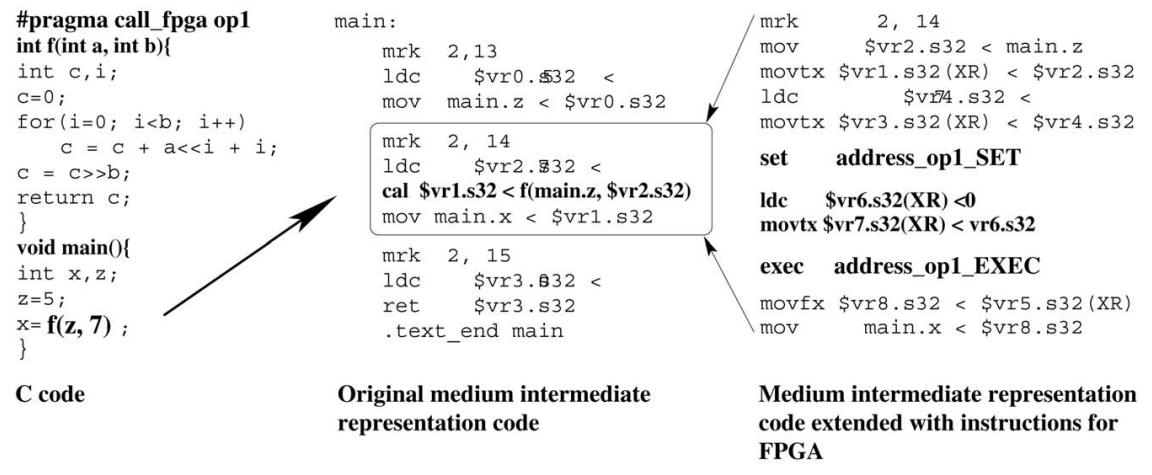

Fig. 6. Medium intermediate representation code.

approaches concerning parallel execution. In case two or more functions considered for CCU implementation do not have any true dependencies, they can be executed in parallel. An example of how this can be performed is depicted in Fig. 7. It should be noted that kernels can, as far as such kernels can, be appropriately transformed to the Molen programming paradigm by: 1) rewriting the kernel as a separate function and 2) defining a clear set of parameters as interface and passing them as values (or references) between the modified "old" and the new function code. All of the communication between the two functions should be done as much as possible via input/ output parameters since both parts will execute in different contexts. The Molen paradigm facilitates modular system design. For instance, hardware implementations described in an HDL (VHDL, Verilog, or System-C) are mappable to any FPGA technology in a straightforward manner. The only requirement is to satisfy the Molen set and execute interface. In addition, a wide set of functionally similar CCU designs (from different providers), e.g., the sum of absolute differences (SAD) or IDCT, can be collected in a database allowing easy design space explorations.

Interrupts and miscellaneous considerations. Our approach is based on the GPP coprocessor paradigm (see, for example, [19], [20]). Consequently, all known coprocessor interrupt techniques [21] are applicable. In order to support the core processor interrupts properly, the following parts are essential for any Molen implementation:

1. Hardware to detect interrupts and terminate the execution before the state of the machine is changed

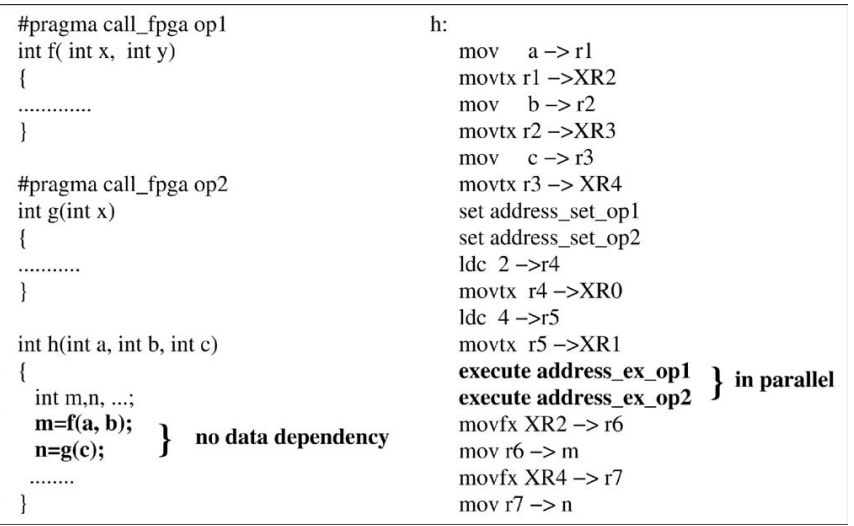

Fig. 7. Parallel execution in Molen. is assumed to be implemented in both the core processor (as usual) and the reconfigurable processor.

2. Interrupt policies, e.g., priorities, are usually handled by the core processor. Consequently, hardware to communicate interrupts to the core processor is implemented in the reconfigurable processor.

3. Initialization (via the core processor) of the appropriate routines for interrupt handling.

The compiler assumption is that the programmer/ implementor of a reconfigurable hardware follows a coprocessor paradigm and that (as in the GPP paradigm) the reconfigurable coprocessor facility can be viewed as an extension of the core processor architecture, the way coprocessors, such as floating-point, vector facilities, etc., have been viewed in conventional architectures.

\section{A MicROARCHITECTURE AND ITS IMPLEMENTATION}

In this section, we discuss issues encountered in implementing a microarchitecture supporting the minimal Molen $\pi$ ISA on the Virtex II Pro with the embedded PowerPC 405 serving as the core processor. Experienced microcode designers will recognize that, for performance reasons, there is the necessity of having microcode that resides permanently in the control store and microcode that is pageable. We borrow a "bit" from the instruction to implement resident/pageable microcode. In the instruction format (see Fig. 8), the location of the microcode is indicated by the resident/pageable-bit (R/P-bit) which implicitly determines the interpretation of the address field, i.e., as a memory address $\alpha(\mathrm{R} / \mathrm{P}=1)$ or as a $\rho$-control store address $\rho \mathrm{CS}-\alpha(\mathrm{R} / \mathrm{P}=0)$ indicating a location within the $\rho \mu$-code unit. This location contains the first instruction of the microcode which must always be terminated, e.g., by an end_op microinstruction.

The $\rho \mu$-code unit. The reconfigurable microcode ( $\rho \mu$-code) unit can be implemented in configurable or fixed hardware. In this section, for simplicity, we assume that the $\rho \mu$-code unit is hardwired. The internal organization of the $\rho \mu$-code unit is depicted in Fig. 9. The $\rho \mu$-code unit is comprised of three main parts: the sequencer, the $\rho$-control store, and the $\rho \mu$-code loading unit. The sequencer mainly determines the microcode execution sequence. The $\rho$-control store is used as a storage facility for microcode. The $\rho \mu$-code loading unit, as its name suggests, is responsible for the loading of reconfigurable microcode from the memory. The 
p-set/c-set/execute

\begin{tabular}{|l|l|l|}
\hline OPC & R/P & $\rho C S-\alpha / \alpha$ \\
\hline \\
$\substack{\text { opcode } \\
\text { resident/pageable } \\
0 / 1}$ & address \\
\hline
\end{tabular}

Fig. 8. The $p$-set, $c$-set, and execute instruction format.

execution of microcode starts with the sequencer receiving an address from the arbiter (see Fig. 3) and interpreting it according to the $\mathrm{R} / \mathrm{P}$-bit. When receiving a memory address, it must be determined whether the microcode is already cached in the $\rho$-control store or not. This is done by checking the residence table (see Fig. 10) which stores the most frequently used translations of memory addresses into $\rho$-control store addresses and keeps track of the validity of these translations. It can also store other information: least recently used (LRU) and possibly additional information required for virtual addressing ${ }^{2}$ support. In the case that a memory address is received and the associated microcode is not present in the $\rho$-control store, the $\rho \mu$-code unit initiates the loading of microcode from the memory into the $\rho$-control store. In case a $\rho \mathrm{CS}-\alpha$ is received or a valid translation into a $\rho \mathrm{CS}-\alpha$ is found, the $\rho \mathrm{CS}-\alpha$ is transferred to the "determine next microinstruction"-block. This block determines which (next) microinstruction needs to be executed:

- When receiving the address of the first microinstruction. Depending on the R/P-bit, the correct $\rho \mathrm{CS}-\alpha$ is selected, i.e., from the instruction field or from the residence table.

- When already executing microcode. Depending on previous microinstruction(s) and/or results from the CCU, the next microinstruction address is determined.

The resulting $\rho \mathrm{CS}-\alpha$ is stored in the $\rho$-control store address register $(\rho \mathrm{CSAR})$ before entering the $\rho$-control store. Using the $\rho \mathrm{CS}-\alpha$, a microinstruction is fetched from the $\rho$-control store and then stored in the microinstruction register (MIR) before it controls the CCU reconfiguration or before it is executed by the CCU. The $\rho$-control store is comprised of two sections, ${ }^{3}$ namely, a set section and an execute section. Both sections are further divided into a fixed part and a pageable part (see Fig. 11). The fixed part stores the resident reconfiguration and execution microcode of the set and execute phases, respectively. Resident microcode is commonly used by several invocations (including reconfigurations) and it is stored in the fixed part so that the performance of the set and execute phases is possibly enhanced. Which microcode resides in the fixed part of the $\rho$-control store is determined by performance analysis of various applications and by taking into consideration various software and hardware parameters. Other microcode is stored in memory and the pageable part of the $\rho$-control store acts like a cache to provide temporal storage. Consequently, cache mechanisms are required to ensure proper $\rho$-control store operation. The residence table

2. For simplicity of discussion, we assume that the system only allows real addressing.

3. Both sections can be identical, but they probably only differ in microinstruction word sizes.

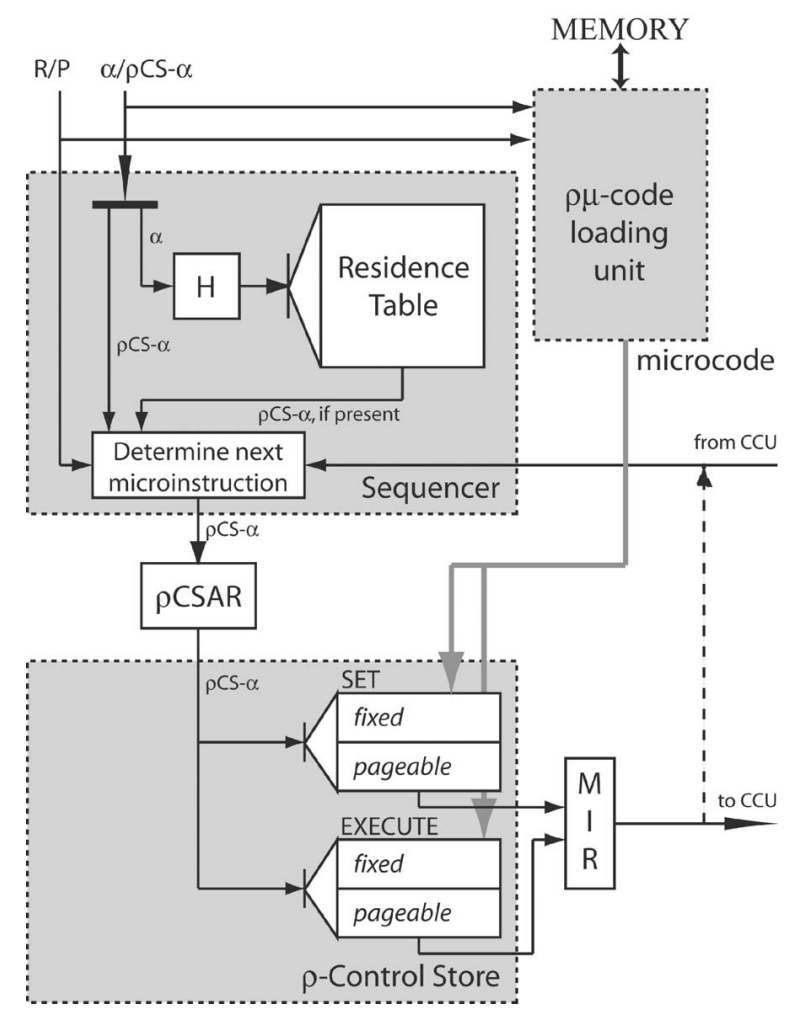

Fig. 9. $\rho \mu$-code unit internal organization.

invalidates entries when microcode has been replaced (utilizing the valid (V) bit) or substitutes the least recently used (LRU) entries with new ones. Finally, the residence table can be separate or common (requiring an additional S/E-bit to allow separation) for both the set and execute pageable $\rho$-control store sections. In the remainder of this section, we present some implementation issues of the minimal Molen $\pi$ ISA utilizing a PowerPC 405 as the core processor, as used in our experimental validation. The minimal $\pi$ ISA consists of the following instructions: set, execute, movtx, and movfx. The arbiter (described in detail in [22]) performs a partial decoding of instructions in order to determine where instructions should be issued. The set and execute instructions will be issued to the reconfigurable processor and, in this specific implementation, the movtx and movfx instructions are issued to the core processor. The latter is due to the fact that both move instructions are

RESIDENCE TABLE

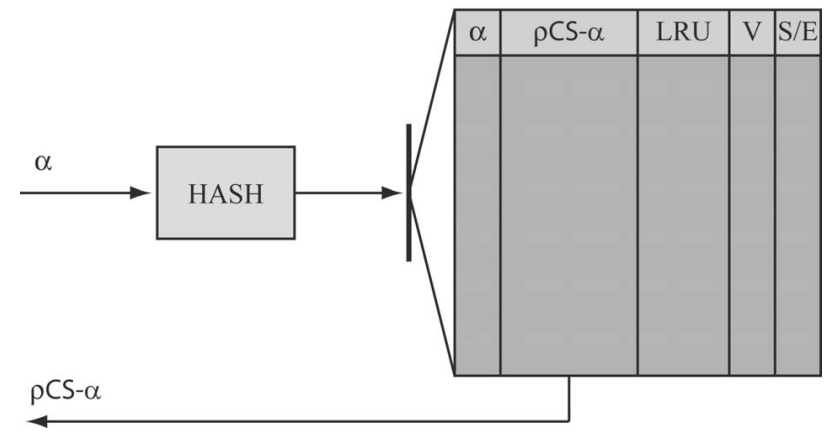

Fig. 10. The sequencer's residence table. 


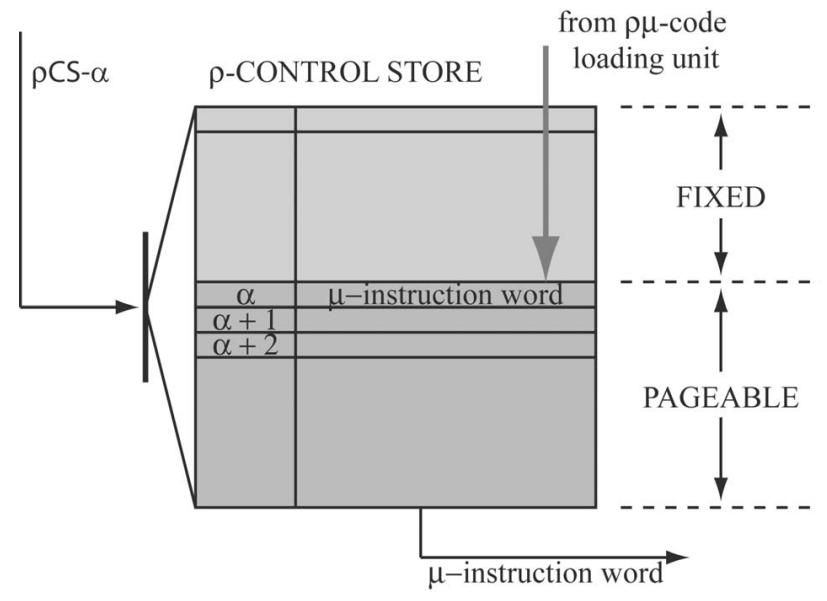

Fig. 11. Internal organization of one section of the $\rho$-control store.

mapped to existing PowerPC instructions, namely, mtdcr and mfdcr, respectively.

General requirements of the arbiter. The arbiter controls the proper coprocessing of the core processor and the reconfigurable processor (see Fig. 3) by directing instructions to either of these processors. It arbitrates the data memory access of the reconfigurable and core processors and it distributes control signals and the starting microcode address to the $\rho \mu$-code unit.

In Fig. 12, a general view of an arbiter organization is depicted. The arbiter operation is based on the decoding of the incoming instructions and either directs instructions to the core processor or generates an instruction sequence to control the state of the core processor. The latter instruction sequence is referred to as "arbiter emulation instructions." Upon decoding of either a set or an execute instruction, the following actions are initiated:

1. Arbiter emulation instructions are multiplexed to the core processor instruction bus and essentially drive the processor into a wait state.

2. Control signals from the decode block are transmitted to the control block in Fig. 12, which performs the following:

a. Redirect the microcode location address to the $\rho \mu$-code unit.

b. Generate an internal code representing either a set or execute instruction (Ex/Set) and delivering it to the $\rho \mu$-code unit.

c. Initiate the reconfigurable operation by generating "start reconf. operation" signal to the $\rho \mu$-code unit.

d. Reserve the data memory control for the $\rho \mu$-code unit by generating a memory occupy signal to the (data) memory controller.

e. Enter a wait state until the signal "end of reconf. operation" arrives.

An active "end of reconf. operation" signal initiates the following actions: 1) Data memory control is released back to the core processor. 2) An instruction sequence is generated to ensure proper exiting of the core processor from the wait state. 3) After exiting the wait state, the program execution

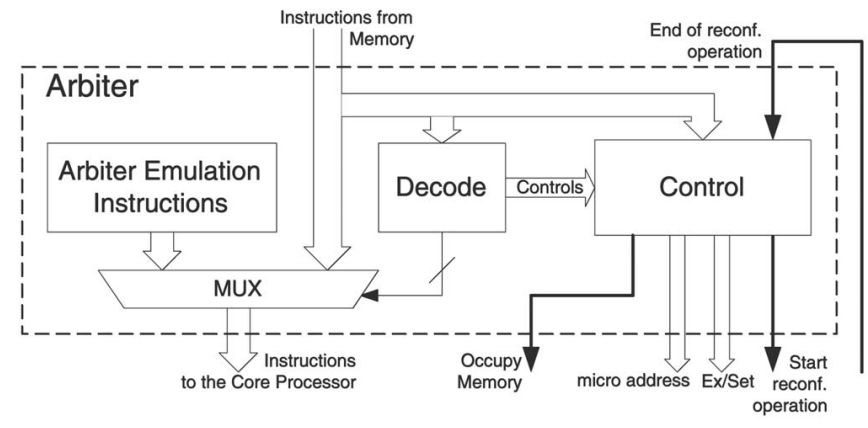

Fig. 12. General arbiter organization.

continues with the instruction immediately following the last executed reconfigurable processor instruction.

Software considerations For performance reasons, PowerPC special operating modes instructions were not used-exiting special operating modes is usually performed by an interrupt. We employed the "branch to link register" (blr) instruction to emulate a wait state and "branch to link register and link" (blrl) instruction to move the processor out of this state. The difference between these instructions is that blrl modifies the link register (LR), while blr does not. The next instruction address is the effective address of the branch target, stored in the link register. When blrl is executed, the new value loaded into the link register is the address of the instruction following the branch instruction. Thus, the arbiter emulation instructions, stored into the corresponding block in Fig. 12, are reduced to only one instruction for wait and one for "wake-up" emulation. The PowerPC architecture allows out-of-order execution of memory and I/O transfers, which has to be taken into account in the implementation. To guarantee that data dependency conflicts do not occur during reconfigurable operation, the PowerPC "synchronization" instruction (sync) can be utilized before a set or execute instruction. In other out-of-order execution architectures, data dependency conflicts should be resolved by specific dedicated features of the target architectures. In in-order architecture implementations, this problem does not exist.

Instruction encoding. Previously, we discussed that the movtx and movfx instructions are mapped to the existing PowerPC instructions mtdcr and $\mathbf{m f d c r}$. This implemented solution is imposed by the fact that the Virtex II Pro PowerPC core has a dedicated interface to the so-called Device Control Registers (DCR) [23] and two instructions that support DCR transfers (namely, $\mathbf{m t d c r}$ and $\mathbf{m f d c r}$ ). It should be noted that this is a PowerPC specific implementation and not applicable in the general case. This leaves only the set and execute instructions to be encoded. We follow the PowerPC I-form and choose unused opcodes for both instructions. The manner to distinguish a set instruction, an execute instruction (using the same opcode), and resident/pageable (R/P) addresses is via instruction modifiers.

Arbiter hardware requirements. To implement the arbiter, we have considered the following: 1) Information, related to instruction decoding, arbitration, and timing is obtained only through the instruction bus (from memory). 2) The PowerPC instruction bus is 64-bit wide and instructions are fetched in couples. 3) Speculative prefetches should not disturb the correct timing of a reconfigurable processor instruction execution. The arbiter for PowerPC 


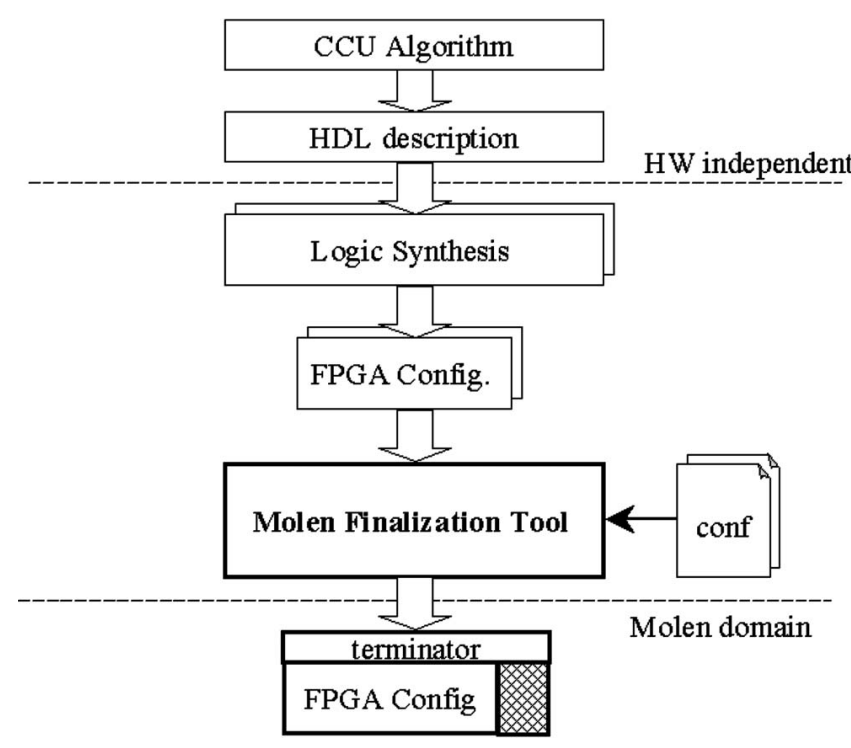

Fig. 13. Molen finalization.

has been described in synthesizable VHDL and mapped on the Virtex II Pro FPGA of Xilinx.

Microcode configuration, termination, and finalization. The FPGA reconfiguration files generated after synthesis contain unpredictable bit patterns and will highly depend on the targeted FPGA technology. It is essential to note that the same high-level HDL description results in completely different configuration bitstreams when different technologies are targeted. In case of execution microcode, the end_op microinstruction at the end of the microcode segment is sufficient for the proper termination of the reconfigurable operation provided that the microcode is properly aligned into the memory. This technique, however, would not work for reconfiguration microcode because the reconfiguration bitstreams are an arbitrary bit sequence. Therefore, it is possible that the reconfiguration microcode loading is terminated earlier by a false end_op microinstruction. One approach to resolve early termination is the following: An additional microcode word may be aligned at the starting address of the microprogram segment. This word may contain either the length of the microprogram or its end address. Since both methods do not differ in either implementation or microcode size, we have arbitrarily selected the latter one in our current implementation. The process of preparing the microcode for its final alignment into the targeted main memory is called microcode finalization. In microcode termination, additional termination information should be explicitly added to the microprogrammable configuration code. The automated process of microcode finalization for Molen indicating the place of the finalization tool in the Molen CCU design process is depicted in Fig. 13. The CCU design, described in HDL, can be targeted to different FPGA technologies. This allows descriptions that can be synthesized to any particular technology utilized by Molen.

The configuration file (indicated as conf) contains information about the Molen organization needed for the reconfiguration microcode finalization. The product of the finalization tool is a binary file ready to be used inside the Molen paradigm and can be a linkable object or a high-level data structure, incorporating the binary information, that can be included directly in a $\mathrm{C}$ project before compilation. It should be noted that the reconfiguration microcode endianness is transparent to the proposed approach and does not require special consideration.

$\rho \mu$-code loading unit implementation. The $\rho \mu$-code loading unit (see Fig. 9) is responsible for loading microprograms from the external memory. The start_op signal (not depicted in Fig. 9) is generated by the arbiter and initiates a reconfigurable operation. The $\rho \mu$-code loading unit sequentially generates the addresses of the microprogram in the main memory and the desired microprogram is loaded into the $\rho$-control store. Once the microprogram is available in the $\rho$-control store, i.e., the end address of the microprogram in the external memory is reached, the sequencer starts the execution of the microcode generating microcode addresses toward the $\rho$ CSAR. We have to note that other parts of the $\rho \mu$-code unit are not discussed as they are essentially memorylike elements with appropriate controls.

\section{Evaluation}

In order to evaluate our proposal, we experimented with the Alpha Data XPL Pro lite development board (ADM-XPL) and the Xilinx Project Navigator ISE 5.1 (Service Pack \#3) design environment. As reconfigurable hardware platform, we used the latest Xilinx xc2vp20 devices (speed grade 5) from the Virtex II Pro family. For our experimentation, we target and profile the MPEG-2 application. As implemented in the platform hardware, partial reconfiguration is severely limited because it is allowed only on fixed frame boundaries (the xc2vp20 incorporates $8,214,624$ bit configuration memory divided into 1,756 frames) with no possibilities for frame reduction. This limits the flexibility on CCU reconfiguration sizes. For our experiments, we reconfigure the device at the system initialization stage. There is an additional platform restriction, namely, the available on-chip block RAM (BRAM) memory of $\mathrm{xc} 2 \mathrm{vp} 20$ is limited to $128 \mathrm{kBytes}$ for both instructions and data. Due to the space limitation, we were unable to run any file, I/O, and operating system calls. As a consequence, we used the profiling information to design the kernels as CCU implementations and estimated the performance gains rather than directly run the entire MPEG-2 application on the Molen processor. Furthermore, the following has been assumed: The parts of the applications which can be implemented on the reconfigurable hardware are isolated in functions. The core processor and the reconfigurable processor do not run concurrently. The operations performed on the reconfigurable processor are sequential (for now, we do not consider potential parallelism due to the lack of compiler support). The applications are compiled without optimizations. The PowerPC processors in VirtexII Pro do not implement floating-point instructions. Therefore, the floating-point data type of the DCT coefficients utilized in the MPEG-2 encoder benchmark has been converted to integer data types. The proper integer arithmetic has been implemented for fairness.

Software Profiling Results. The first step involves identifying the functions that are most suitable for hardware implementation. For this purpose, we performed the measurements on a PowerPC 970 running at 1,600 MHz. The considered applications are a set of multimedia benchmarks consisting of the Berkeley implementation of 
TABLE 1

MPEG2 Profiling Results for Each of the Considered Functions and Its Descendants

\begin{tabular}{|c|c|c|c|c|c||c|}
\multicolumn{9}{|c}{ MPEG2 encoder } & MPEG2 decoder \\
\hline sequence & \#frames@Resolution & SAD (16 x 16) & DCT $(8 \times 8)$ & IDCT $(8 \times 8)$ & Total & IDCT $(8 \times 8)$ \\
\hline carphone & $96 @ 176 \times 144$ & $51.1 \%$ & $12.5 \%$ & $1.3 \%$ & $64.9 \%$ & $50.4 \%$ \\
\hline claire & $168 @ 360 \times 288$ & $53.8 \%$ & $11.8 \%$ & $1.0 \%$ & $66.6 \%$ & $37.6 \%$ \\
\hline container & $300 @ 352 \times 288$ & $56.2 \%$ & $10.7 \%$ & $1.0 \%$ & $67.9 \%$ & $40.4 \%$ \\
\hline tennis & $112 @ 352 / 240$ & $60.0 \%$ & $9.5 \%$ & $0.8 \%$ & $70.3 \%$ & $40.5 \%$ \\
\hline
\end{tabular}

the MPEG-2 encoder and the MPEG-2 decoder included in libmpeg2. The objective is to identify the most timeconsuming operations among the following operations, namely, SAD (sum of absolute-difference), 2D-DCT (2-dimensional discrete cosine transform) and 2D-IDCT (2-dimensional inverse DCT). As input data, we used a representative series of video sequences consisting of frames with varying resolutions, presented in Table 1, column two.

For our measurements, we used the GNU profiler gprof to determine the amount of time spent in each function and its descendants. The results for the considered benchmarks, input data and operations are presented in Table 1. For the MPEG2 encoder application, we notice that the SAD function consumes more than 50 percent of the application time (Table 1, column three) and, consequently, it is the best candidate for hardware implementation. The integer DCT function accounts for around 11 percent of the application time (Table 1, column four). For the IDCT function, we notice that, although in the MPEG2 encoder it takes only around 1 percent of the application time (Table 1, column five), in the MPEG2 decoder it requires, on average, 42 percent of the application time (Table 1, column seven). The total execution time spent in the SAD, DCT, and IDCT operations in the MPEG2 encoder (presented in Table 1, column six) emphasizes that these functions require around $2 / 3$ of the total application time. Consequently, all considered functions are good candidates for hardware implementations, although their contribution to the performance improvement may differ per application.

Molen organization synthesis results. The Molen organization has been described in VHDL and simulated with Modeltech's ModelSim SE 5.7c. The synthesis has been performed with Project Navigator ISE 5.2 SP3 from Xilinx and the Virtex II Pro has been considered as a target reconfigurable technology. For the prototype implementation, we have considered a microcode word length of 64 bits. A 32 MByte memory segment has been considered for storing microprograms into a 64-bit organized main memory. The $\rho$-control store has been designed to handle up to $8 \mathrm{KBytes}$ of 64 -bit microcode words. As primary microcode storage units for the $\rho$-control store, we have used the BRAM blocks of the FPGA fabric, configured as a dual port memory. Each port is unidirectional—a read-only port is used to feed the MIR, while a write-only one loads microcodes from the external memory into the pageable section of the $\rho$-control store. The XREGs have been implemented in a single BRAM organized as $512 \times 32$-bit storage. Hardware costs reported by the synthesis tools are presented in Table 2. The first column presents the FPGA resources considered. Column two reports the actual values of these resources, consumed by the reconfigurable processor, without considering any CCU implementation, i.e., the $\rho \mu$-code unit and the associated infrastructure. This includes the $\rho \mu$-code loading unit, the sequencer, and the $\rho$-control store. Column three presents resource utilization of the arbiter. In column four, the resources consumed by the entire Molen organization are displayed, including the reconfigurable processor infrastructure, the arbiter, and the XREGs. Finally, columns five and six, respectively, present the available FPGA resources in the xc2vp20 chip and the utilized part of these resources by the Molen organization (in percentages). The results strongly suggest that the Molen infrastructure consumes trivial hardware resources, leaving almost the entire area for CCU implementation.

Synthesis results of the CCU implementations. We implemented the functionalities of the kernels, suggested by the profiling results, into reconfigurable hardware. Synthesis results for the xc2vp20 chip are reported in Table 3.

For the SAD function, we implemented the organization proposed in [24]. The super-pipelined 16-byte version of this SAD organization (SAD16) is capable of processing one 16-pixel line (1 pixel is 1 byte) of a macroblock in 17 cycles at over $300 \mathrm{MHz}$. The 128-byte version (SAD128) processes eight macroblock lines in 23 cycles and the 256-byte version (SAD256), processes an entire $16 \times 16$-pixel macroblock in 25 cycles at $300 \mathrm{MHz}$. The latter design (SAD256) requires more resources than available in the xc2vp20 chip used for this experimentation; therefore, we consider it for future implementation when the larger xc2vp50 becomes available. To support the DCT and IDCT kernels, we synthesized the 2-D DCT and 2D-IDCT v.2.0 cores available as IPs in the

TABLE 2

Molen Organization Synthesis Results

\begin{tabular}{|l|r|r|r|r|r|}
\hline Device xc2vp20 & RP* & Arbiter & $\begin{array}{r}\text { Total incl. } \\
\text { XREGs }\end{array}$ & $\begin{array}{r}\text { Available } \\
\text { Resources }\end{array}$ & $\%$ \\
\hline \# Slices & 71 & 84 & 156 & 10304 & 1 \\
\# Flip Flops & 78 & 69 & 147 & 20608 & 1 \\
\# 4 inp LUTs & 171 & 150 & 322 & 20608 & 1 \\
\# BRAMs: & 4 & N.A. & 5 & 112 & 3 \\
$f_{\text {max }}$ [MHz] & 130 & 143 & 130 & N.A. & N.A. \\
\hline
\end{tabular}

* Reconfigurable processor without any CCU implemented 
TABLE 3

Synthesis Results per CCU Implementation

\begin{tabular}{|l|r|r|r|r|r|r|}
\hline $\begin{array}{l}\text { Device } \\
\text { xc2vp20-5 }\end{array}$ & $\begin{array}{r}\text { SAD } \\
16\end{array}$ & $\begin{array}{r}\text { SAD } \\
128\end{array}$ & $\begin{array}{r}\text { SAD } \\
256^{*}\end{array}$ & DCT & IDCT & $\begin{array}{r}\text { Available } \\
\text { Resources }\end{array}$ \\
\hline \# Slices & 831 & 6807 & $13613^{*}$ & 4314 & 5436 & 10304 \\
\# Flip Flops & 1448 & 11862 & $23724 *$ & 7964 & 9876 & 20608 \\
\# 4 inp LUT & 1390 & 11379 & $22757^{*}$ & 6832 & 8624 & 20608 \\
\# BRAMs: & N.A. & N.A. & N.A. * & 2 & 2 & 112 \\
$f_{\max }[\mathrm{MHz}]$ & 310 & 310 & $310^{*}$ & 96 & 96 & N.A. \\
\hline
\end{tabular}

* Results for xc2vp50 FPGA

Xilinx Core Generator Tool. The parameters for their synthesis are presented in Table 4.

Since the recommended maximum PowerPC frequency for the xc2vp20-5 FPGA is $250 \mathrm{MHz}$, the ADM-XPL prototyping board vendors recommend obtaining this frequency from a user clock of $83 \mathrm{MHz}$ multiplied by 3 using the on-chip FPGA Digital clock managers (DCMs). Considering these recommendations and synthesis results from Table 3 for our experiments, we have to run the DCT and IDCT functions at a frequency three times lower than the PowerPC clock. The SAD designs were clocked at the same frequency as the PowerPC.

MPEG-2 performance experiments. We have embedded the considered CCU implementations within the Molen organization and executed the corresponding software kernels for performance measurements. For our experiments, we first compiled the software kernels for the original PowerPC ISA and ran them on one of the PowerPC405 processors, embedded in the xc2vp20 device. The kernels have been extracted from the original application source code (the ANSI C code used for the profiling) without any further code modifications. For our experiments, we considered the same data sequences as used in the profiling phase. The PowerPC timers are initialized before a kernel is executed and are read immediately after the kernel execution has completed. Thus, the exact number of PowerPC cycles required for the entire kernel execution can be obtained. After we derived the cycle counts for the PowerPC ISA software runs, we initiated the next stage of the experimentation. At this stage, similar to the code transformation discussed in Section 2, the kernel software code is substituted with a new piece of code to support the $\pi$ ISA. The corresponding kernel CCU configuration is present in the reconfigurable processor considering the

TABLE 4

Synthesis Parameters for the 2-D DCT and 2-D IDCT IPS

\begin{tabular}{|l|r|r|}
\hline Parameter & 2-D DCT & 2-D IDCT \\
\hline Data width [bits] & 16 (signed) & 16 (signed) \\
Coeff. width [bits] & 24 & 24 \\
Result width [bits] & 16 (rounded) & 16 (rounded) \\
cycles/input sample & 6 & 8 \\
Internal latency [cyc] & 94 & 97 \\
\hline
\end{tabular}

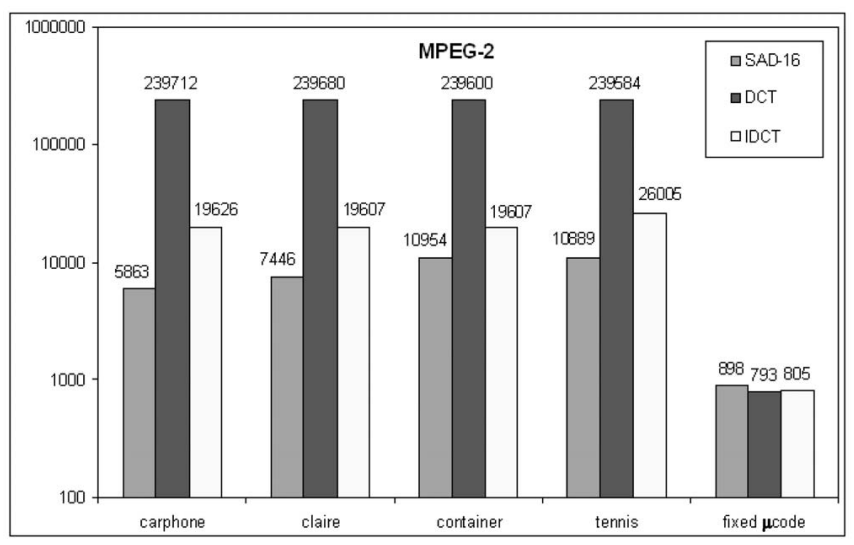

Fig. 14. Cycle numbers for kernels execution in original PowerPC ISA and fixed microcode in $\pi$ ISA.

discussion in the beginning of this section. Identically to the preceding experimentation stage, we obtain the exact number of PowerPC cycles required to complete the entire kernel operation on Molen. The measurements include cycle numbers for transferring parameters to/from the exchange registers (implemented as DCRs), cycles for memory transfers, and data processing cycles. Fig. 14 depicts the measured cycles obtained in the latter two experimentation phases. The first four chart groups present cycle counts for the original PowerPC ISA. The last chart group presents the cycle numbers, consumed by Molen while processing the same data. It should be noted that the performance of the PowerPC software implementations of the three kernels is highly dependent on the data contents. On the contrary, for all four data sequences, the cycle number for the Molen implementation depends only on the amount of data and not on the data contents due to the data independent CCU designs. Therefore, only a single group of results for all data sequences in the Molen execution is presented in Fig. 14. In this figure, only fixed microcode implementations are depicted.

In addition, we have implemented both fixed and pageable microcode implementations for SAD16 and SAD128. Table 5 reports measured cycle numbers for executing the SAD kernel over a single macroblock in different Molen configurations. As has been noted, the SAD256 implementation hardware requirements exceed the capabilities of the xc2vp20 device we used. Therefore, the corresponding SAD256 cycle numbers in Table 5 have been extrapolated from the results of SAD16 and SAD128.

After the cycle numbers for the execution of each kernel have been obtained, both for PowerPC and Molen, the speedup of each kernel can be estimated. Table 6 presents the calculated speedups for each of the considered data sequences with respect to each CCU implementation.

Projected application speedup. Results in Table 6 suggest that the considered kernels can be speeded up to 300 times and one can incorrectly assume that the entire application can be speeded up to the same orders of magnitude. ${ }^{4}$ In the following, we are going to prove theoretically, combined with experiments, that, in fact,

4. If the considered kernels are the entire application, speedups of the same orders of magnitude can be expected. If this is not the case, as in the considered MPEG-2, the above assumption is incorrect. 
TABLE 5

Cycles per Macroblock for Different SAD Implementation

\begin{tabular}{|l|c|c|c|}
\hline & SAD16 & SAD128 & SAD256 \\
\hline fixed microcode & 898 & 311 & 264 \\
peageable microcode & 914 & 331 & 284 \\
\hline
\end{tabular}

lower, yet considerable and impressive for the GPP domain, overall application speedups could be expected. As indicated earlier, due to space limitations, no file, I/O, or operating system calls have been implemented on the prototype FPGA, thus the application speedup can only be estimated. To calculate the projected speedup of the entire application with respect to the CCU implementations and the $\pi \mathrm{ISA}$, we employed the well-known Amdahl's law, utilizing the following notations: Let us assume $T$ to be the execution time of the original program (say measured in cycles) and $T_{S E i}$ - time to execute kernel $i$ in software, which we would like to speed up in reconfigurable hardware. Assume $T_{\rho i}$ is the execution time (in $\pi \mathrm{ISA}$ ) for the reconfigurable implementation of kernel $i$. Assuming $a_{i}=\frac{T_{S E i}}{T}$ and $s_{i}=\frac{T_{S E i}}{T_{p i}}$, the speedup of the program with respect to the reconfigurable implementation of kernel $i$ is:

$$
S_{i}=\frac{T}{T-T_{S E i}+T_{\rho i}}=\frac{1}{1-\left(a_{i}-\frac{a_{i}}{s_{i}}\right)} .
$$

Identically, assuming $a=\sum_{i} a_{i}$, all the kernels considered for reconfigurable implementation would speed up the program with:

$$
S=\frac{1}{1-\left(a-\sum_{i} \frac{a_{i}}{s_{i}}\right)}, S_{\max }=\lim _{\forall s_{i} \rightarrow \infty} S=\frac{1}{1-a},
$$

where $S_{\max }$ is the theoretical maximum speedup. Parameters $a_{i}$ are the profiling results from Table 1 and parameters $s_{i}$ are the results from Table 6 . The projected overall speedup figures for the entire MPEG-2 encoder and MPEG-2 decoder applications are reported in Table 7. Columns labeled "theory" present the theoretically achievable maximum speedup calculated with respect to (2). Columns labeled with "impl." contain data for the projected speedups with respect to the considered Molen implementation. For the MPEG-2 encoder, the simultaneous configuration of the SAD128, DCT, and IDCT operations employing fixed microcode implementations has been considered. For the MPEG-2 decoder, only the IDCT reconfigurable implementation has been employed. Columns with label "imp./th." in Table 7 indicate (in percentages) how close the real speedup is to the theoretically attainable one. Reported results strongly suggest that the actual speedup of the MPEG-2 encoder and decoder obtained during our practical experimentation very closely approach the theoretically estimated maximum possible speedups.

\section{ConcLusions}

In this paper, we presented a polymorphic processor paradigm that allows the programmer/designer to modify and extend the processor functionality and hardware at will without architectural and design modifications. The
TABLE 6

MPEG-2 Speedup Estimations for Different Kernels

\begin{tabular}{|l|c|c|c|c|c|c|c|c|}
\hline \multirow{2}{*}{} & \multicolumn{2}{|c|}{ SAD16 } & \multicolumn{2}{c|}{ SAD128 } & \multicolumn{2}{c|}{ SAD256 } & DCT & IDCT \\
\cline { 2 - 8 } & fixed & pag. & fixed & pag. & fixed & pag. & fixed & fixed \\
\hline carph. & 6.5 & 6.4 & 18.9 & 17.7 & 22.2 & 20.6 & 302.3 & 24.4 \\
claire & 8.3 & 8.1 & 23.9 & 22.5 & 28.2 & 26.2 & 302.2 & 24.4 \\
cont. & 12.2 & 12.0 & 35.2 & 33.1 & 41.5 & 38.6 & 302.1 & 24.4 \\
tennis & 12.1 & 11.9 & 35.0 & 32.9 & 41.2 & 38.3 & 302.1 & 32.3 \\
\hline
\end{tabular}

TABLE 7

Overall Speedup Estimations for the Entire MPEG2

\begin{tabular}{|l|c|c|c|c|c|c|}
\hline \multirow{2}{*}{} & \multicolumn{3}{|c|}{ MPEG2 encoder* } & \multicolumn{3}{c|}{ MPEG2 decoder } \\
\cline { 2 - 7 } & theory & impl. & impl./th. & theory & impl. & impl./th. \\
\hline carphone & 2.85 & 2.64 & $93 \%$ & 2.02 & 1.94 & $96 \%$ \\
claire & 2.99 & 2.80 & $94 \%$ & 1.60 & 1.56 & $98 \%$ \\
container & 3.12 & 2.96 & $95 \%$ & 1.68 & 1.63 & $97 \%$ \\
tennis & 3.37 & 3.18 & $94 \%$ & 1.68 & 1.65 & $98 \%$ \\
\hline
\end{tabular}

* fixed $\rho \mu$-code SAD128 + DCT + IDCT

proposal solves a number of limitations of existing approaches, such as the opcode space explosion, and it requires only a one time extension of the instruction set to incorporate an almost unlimited number of reconfiguration functions per single programming space. Finally, it introduces a modular approach allowing easy porting of applications to different reconfigurable platforms and allows compiler controlled parallelism.

\section{ACKNOWLEDGMENTS}

This research is partially supported by PROGRESS, the embedded systems research program of the Dutch organization for Scientific Research NWO.

\section{REFERENCES}

[1] G. Blaauw and F. Brooks Jr., Computer Architecture. AddisonWesley, 1997

[2] M. Sima, S. Vassiliadis, S. Cotofana, J. van Eijndhoven, and K. Vissers, "Field-Programmable Custom Computing Machines-A Taxonomy," Proc. 12th Int'l Conf. Field Programmable Logic and Applications (FPL), pp. 79-88, Sept. 2002.

[3] K. Compton and S. Hauck, "Reconfigurable Computing: A Survey of Systems and Software," ACM Computing Surveys, vol. 34, no. 2, pp. 171-210, 2002

[4] S. Hauck, T. Fry, M. Hosler, and J. Kao, "The Chimaera Reconfigurable Functional Unit," Proc. IEEE Symp. Field-Programmable Custom Computing Machines, pp. 87-96, 1997.

[5] A.L. Rosa, L. Lavagno, and C. Passerone, "Hardware/Software Design Space Exploration for a Reconfigurable Processor," Proc. Design, Automation, and Test in Europe Conf. (DATE), pp. 570-575, 2003.

[6] M. Gokhale and J. Stone, "Napa C: Compiling for a Hybrid RISC/ FPGA Architecture," Proc. IEEE Symp. Field-Programmable Custom Computing Machines, pp. 126-135, Apr. 1998.

[7] F. Campi, M. Toma, A. Lodi, A. Cappelli, R. Canegallo, and R. Guerrieri, "A VLIW Processor with Reconfigurable Instruction Set for Embedded Applications," Proc. Int'l Solid State Circuits Conf. (ISSCC) Digest of Technical Papers, pp. 250-251, Feb. 2003. 
[8] A. Ye, N. Shenoy, and P. Banerjee, "A C Compiler for a Processor with a Reconfigurable Functional Unit," Proc. ACM/SIGDA Symp. FPGAs, pp. 95-100, 2000.

[9] J. Becker and R. Hartenstein, "Configware and Morphware Going Mainstream," J. Systems and Architecture, vol. 49, nos. 4-6, pp. 127142, 2003.

[10] A. Turjan, T. Stefanov, B. Kienhuis, and E. Deprettere, "The Compaan Tool Chain: Converting Matlab into Process Networks," Proc. Designer's Forum of Design, Automation, and Test in Europe Conf. 2002, pp. 258-264, 2002.

[11] S. Vassiliadis, S. Wong, and S. Cotofana, "Microcode Processing: Positioning and Directions," IEEE Micro, vol. 23, no. 4, pp. 21-30, July/Aug. 2003.

[12] J.M.P. Cardoso and H.C. Neto, "Compilation for FPGA-Based Reconfigurable Hardware," IEEE Design and Test of Computers, vol. 20, no. 2, pp. 65-75, Mar./Apr. 2003.

[13] C. Zissulescu, T. Stefanov, B. Kienhuis, and E. Deprettere, "LAURA: Leiden Architecture Research and Exploration Tool," Proc. 13th Int'l Conf. Field Programmable Logic and Applications (FPL), pp. 911-920, Sept. 2003.

[14] S. Vassiliadis, S. Wong, and S. Cotofana, "The MOLEN $\rho \mu$-Coded Processor," Proc. 11th Int'l Conf. Field Programmable Logic and Applications (FPL), pp. 275-285, Aug. 2001.

[15] S. Vassiliadis, G. Gaydadjiev, K. Bertels, and E. Moscu Panainte, "The Molen Programming Paradigm," Proc. Third Int'l Workshop Systems, Architectures, Modeling, and Simulation, pp. 1-7, July 2003.

[16] E. Moscu Panainte, K. Bertels, and S. Vassiliadis, "Compiling for the Molen Programming Paradigm," Proc. 13th Int'l Conf. Field Programmable Logic and Applications (FPL), pp. 900-910, Sept. 2003.

[17] http://suif.stanford.edu/suif/suif2, 2004.

[18] http://www.eecs.hardvard.edu/hube/research/machsuif.html, 2004.

[19] A. Padegs, B. Moore, R. Smith, and W. Buchholz, "The IBM System/370 Vector Architecture: Design Considerations," IEEE Trans. Computers, vol. 37, pp. 509-520, 1988.

[20] W. Buchholz, "The IBM System/370 Vector Architecture," IBM Systems J., vol. 25, no. 1, pp. 51-62, 1986.

[21] M. Moudgill and S. Vassiliadis, "Precise Interrupts," IEEE Micro, vol. 16, no. 1, pp. 58-67, Jan. 1996.

[22] G. Kuzmanov and S. Vassiliadis, "Arbitrating Instructions in an $\rho \mu$-Coded CCM," Proc. 13th Int'l Conf. Field Programmable Logic and Applications (FPL '03), pp. 81-90, Sept. 2003.

[23] Virtex-II Pro Platform FPGA Handbook. Xilinx Corp., 2002.

[24] S. Vassiliadis, E. Hakkennes, S. Wong, and G. Pechanek, "The Sum-of-Absolute-Difference Motion Estimation Accelerator," Proc. 24th Euromicro Conf., pp. 559-566, Aug. 1998.

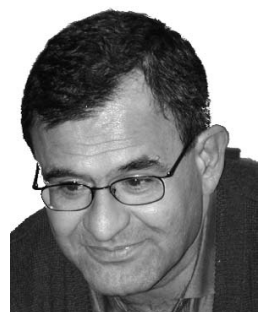

Stamatis Vassiliadis is currently a chair professor in the Electrical Engineering Department at Delft University of Technology (TU Delft), The Netherlands. He had also served on the Electrical Engineering Faculties of Cornell University, Ithaca, New York, and the State University of New York, Binghamton. He worked for a decade with IBM, where he was involved in a number of advanced research and development projects. For his work, he received numerous awards, including 24 publication awards, 15 invention awards, and an outstanding innovation award for engineering/scientific hardware design. His 70 US patents rank him as the top all-time IBM inventor. In 1992, he received an honorable mention best paper award from the ACM/IEEE MICRO25. He received the best paper awards from the IEEE CAS (1998, 2002), IEEE ICCD (2001), and PDCS (2002). He is an IEEE fellow and a member of the IEEE Computer Society.

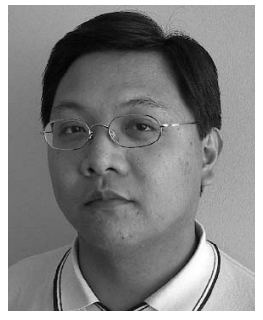

Stephan Wong received the $\mathrm{PhD}$ degree from the Electrical Engineering Department at Delft University of Technology (TU Delft), The Netherlands. He is currently working as an assistant professor in the Computer Engineering Laboratory at TU Delft. He has considerable experience in the design of embedded media processors. $\mathrm{He}$ has also worked on microcoded FPGA complex instruction engines and the modeling of parallel processor communication networks. His research interests include embedded systems, multimedia processors, complex instruction set architectures, reconfigurable and parallel processing, microcoded machines, and network processors. He is a member of the IEEE and the IEEE Computer Society.

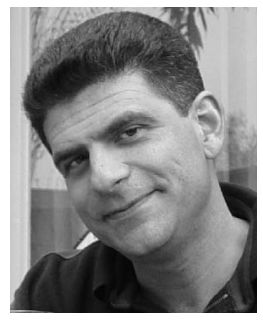

Georgi Gaydadjiev is currently an assistant professor in the Computer Engineering Laboratory, Delft University of Technology, The Netherlands. His research and development experience includes 15 years in hardware and software design at System Engineering Ltd. in Pravetz, Bulgaria, and Pijnenburg Microelectronics and Software B.V. in Vught, The Netherlands. His research interests include embedded systems design, advanced computer architectures, hardware/software codesign, VLSI design, cryptographic systems, and computer systems testing. He is a member of the IEEE and the IEEE Computer Society.

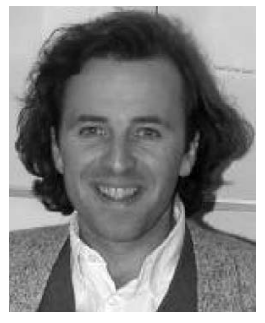

Koen Bertels is currently a member of the faculty of electrical engineering at Delft University of Technology (TU Delft), The Netherlands. His research involves the development of semiautomatic platforms for the design of embedded systems. The tools are intended for $\mathrm{SoC}$ reconfigurable technologies. He is further involved in the simulation and analysis of interacting migrating processes and multiagent systems from a computer engineering perspective. His research interests are in complex systems, reconfigurable computing, agent technology, back-end compilers, semiautomatic tool platforms, and distributed computing. He is a member of the IEEE and the IEEE Computer Society.

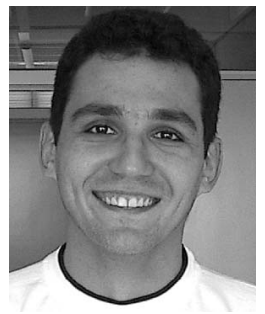

Georgi Kuzmanov received the MSc degree in computer engineering from the Technical University of Sofia, Bulgaria, in 1998 and is currently working toward the $\mathrm{PhD}$ degree at the Computer Engineering Lab at Delft University of Technology (TU Delft), The Netherlands. Between 1998 and 2000, he was with "Info MicroSystems" Ltd., Sofia, where he was involved in several reconfigurable computing and ASIC projects as a research and development engineer. $\mathrm{He}$ is a student member of the IEEE and the IEEE Computer Society. His current research interests include reconfigurable computing, video and image processing, multimedia embedded systems, computer arithmetic, computer architecture, and computer organization.

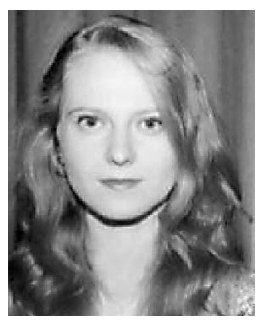

Elena Moscu Panainte received the MSc degree (in computer science) from the "Politehnica" University of Bucharest, Romania, in 2001. Currently, she is a PhD student in the Computer Engineering Laboratory, Electrical Engineering Department, Delft University of Technology, The Netherlands. Her research interests include compiler design, reconfigurable computing, and hardware-software codesign.

$\triangleright$ For more information on this or any other computing topic, please visit our Digital Library at www.computer.org/publications/dlib. 